\title{
Sistema híbrido fotovoltaico (FV) con interacción a la red para zonas rurales de Colombia
}

\section{(PV) photovoltaic hybrid system with interaction to the network to rural areas of Colombia}

\author{
Sistema híbrido fotovoltaico (FV) com rede de interação \\ para a Colômbia rural
}

\author{
Iván Mauricio Ostos Rojas ${ }^{1}$, Carlos Andrés Collazos Morales², \\ Hermes Enrique Castellanos Acuña ${ }^{3}$, Claudia Patricia Fernández Arévalo ${ }^{4}$ \\ ${ }^{1}$ Ingeniero de Recursos Hídrícos y Gestión Ambiental, Master of Engineering. 2Físico, Magister en Ingeniería \\ Electrónica y de Computadores, Doctor en Ciencias, Posdoctor en Tecnología Avanzada ${ }^{3}$ ngeniero Electrónico, \\ Magister en Ingeniería. ${ }^{4}$ Ingeniera Química, Especialista en Ingeniería de Producción. \\ 1,2,3,4 Grupo de Ciencias Básicas y Laboratorios. Universidad Manuela Beltrán. Bogotá. Colombia \\ 1'mostos@b2green.co, ${ }^{2}$ cacollazos@gmail.com, ${ }^{3}$ hsajca@gmail.com, ${ }^{4}$ clapafer@yahoo.com
}

\section{Resumen}

El suministro de energía eléctrica en el municipio de Arbeláez, Cundinamarca, Colombia, presenta interrupciones frecuentes, además de fluctuaciones considerables de voltaje, reduciendo confiabilidad y comodidad del usuario. Colombia cuenta con una localización privilegiada con relativamente constante irradiación solar a lo largo del año, permitiendo la implementación de sistemas fotovoltaicos para generar electricidad como respuesta válida a los problemas presentados por el suministro de energía eléctrica de baja calidad de la red. Los sistemas FV pueden generar energía eléctrica de calidad, sin desviaciones de tensión considerable, evitando inconformidad en el usuario, mala operación de equipos, pérdidas energéticas y pérdidas económicas por averías a equipos expuestos por largos periodos a energía de baja calidad. Se implementa un sistema FV que generará energía de óptima calidad y constante para una casa de descanso. El sistema instalado se programó para trabajar de forma híbrida, la energía proveniente de las celdas solares es la fuente principal de energía para alimentar la demanda, si es requerido y el banco de baterías alcanza un 50\% de descarga, la red eléctrica entraría a trabajar como sistema de respaldo. La generación actual del sistema es de 19.75kWh día, esto representa una reducción total desde diciembre de 2015 hasta agosto de 2016 de $982 \mathrm{~kg}$ de $\mathrm{CO}_{2 \text { eq }}$, con un factor de emisión de $0.18259 \mathrm{kgCO}_{2 e q}$ por cada $\mathrm{kWh}$ comprado de la red en el área. Cada kg de $\mathrm{CO}_{2 \text { eq }}$ no emitido a la atmósfera, es un kg que no contribuirá al aumento del calentamiento global, aportando a la sostenibilidad.

Palabras claves: calentamiento global, Colombia, energías renovables, monitoreo remoto, solar 


\section{Abstract}

The supply of electricity in the municipality of Arbelaez, Cundinamarca, Colombia, has frequent interruptions, in addition to considerable voltage fluctuations, reducing reliability and user comfort. Colombia boasts a privileged location with relatively constant sunlight throughout the year, allowing the implementation of photovoltaic systems to generate electricity as a valid response to the problems presented by the low quality of network electricity supply. PV systems can generate electric power quality, without deviations of considerable stress, avoiding discomfort on the user, poor operation of equipment, energy losses and economic losses due to spoilage exposed for long periods to low quality power PCs. Implements a PV system that will generate energy for optimal quality and constant for a rest house. The installed system is programmed to work is a hybrid, energy from the solar cells is the main source of energy to feed the demand, if it is required and the battery bank achieves a $50 \%$ discharge, electrical network would go to work as a backup system. The current generation of the system is $19.75 \mathrm{kWh}$ day, this represents a total reduction from December 2015 to August $2016982 \mathrm{~kg} \mathrm{CO}{ }_{\mathrm{e}} \mathrm{q}$, with an emission factor of $0.18259 \mathrm{kgCO} 2_{\mathrm{e}}$ q per each purchased kWh of the network in the area. Each $\mathrm{kg}$ $\mathrm{CO}_{\mathrm{e}} \mathrm{q}$ not emitted to the atmosphere, is a $\mathrm{kg}$ that will not contribute to the increase in global warming, contributing to sustainability.

Key-words: global warming, Colombia, renewable energies, remote monitoring, solar

\section{Resumo}

O fornecimento da energia elétrica na localidade de Arbeláez, Cundinamarca, Colômbia, apresenta interrupções frequentes além de flutuações importantes de voltagem, reduzindo a confiabilidade e o conforto do usuário. Colômbia encontra-se localizada privilegiadamente, com irradiação solar continua ao longo do ano, permitindo a incorporação de sistemas fotovoltaicos para gerar eletricidade de baixa qualidade de rede. Os sistemas FV podem gerar energia elétrica de qualidade sem desvios de tensão evitando o inconformismo do usuário, manejo errado dos equipamentos, perdas energéticas e económicas por problemas em equipamentos expostos a longas jornadas de energia de baixa qualidade. Foi feita a incorporação de um sistema FV que vai gerar energia de ótima qualidade e continua para uma casa de descanso. O sistema instalado foi programado para trabalhar de forma híbrida, a energia que vem de células solares é a principal fonte de energia para alimentar a demanda, se for requerido e o banco de baterias atinge um descarregamento do $50 \%$, a rede elétrica começa a trabalhar como sistema de respaldo. A geração atual do sistema é de $19.75 \mathrm{kWh}$ dia, isso representa uma redução total desde dezembro de 2015 até agosto de 2016 de $982 \mathrm{~kg}$ de $\mathrm{CO}_{2 e q}$, com fator de emissão de $0.18259 \mathrm{~kg}$ $\mathrm{CO}_{2 \text { eq }}$ por cada kWh comprado da rede na área. Cada kg de $\mathrm{CO}_{2 \text { eq }}$ não emitido à atmosfera, é um kg que não contribuirá no aumento do aquecimento global, aportando à sustentabilidade.

Palavras-chave: aquecimento global, Colômbia, energias renováveis, monitoramento remoto, solar

\section{Introducción}

La implementación de sistemas fotovoltaicos se puede presentar como una alternativa eficiente y eficaz para suplir la demanda eléctrica en zonas rurales donde la calidad de energía eléctrica suministrada por la red no es óptima por el hecho de presentar interrupciones en el servicio y desviaciones significativas de tensión (OCCIDENTE, 2004; Plangklang, Thanomsat \& Phuksamak, 2016;
Rodríguez, 2008). Dichos sistemas permiten generar electricidad de forma segura y confiable, con la posibilidad de determinar rangos de tensión, corriente y frecuencia requeridos, para así obtener energía de óptima calidad; incrementar la eficiencia de operación de los equipos alimentados y reducir emisiones de Gases de Efecto de Invernadero -GEl-, ya que la fuente de generación eléctrica 
no presenta emisiones en su fase operativa (Hou et al., 2016; Nakagami, Ishihara, Sakai \& Tanaka, 2003). El servicio de suministro de energía eléctrica en el municipio de Arbeláez Cundinamarca, Colombia, presenta interrupciones frecuentes, además de fluctuaciones considerables de voltaje. La calidad de energía eléctrica no óptima presente en el municipio resulta en la reducción del nivel de confiabilidad y comodidad del usuario, y de la eficiencia de los equipos eléctricos conectados a la red (OCCIDENTE, 2004).

Mediante esta investigación se buscó implementar un sistema FV que generara energía de calidad óptima y constante para una casa de descanso localizada en municipio de Arbeláez, y así suplir la demanda presentada por el usuario que es de $28 \mathrm{kWh}$, el sistema contará con 30 paneles solares policristalinos de $255 \mathrm{Wp}$ cada uno; banco de baterías, permitiendo al usuario la posibilidad de permanecer desconectado de la red; controladores tipo MPPT --rastreo del máximo punto de generación-, para mayor eficiencia; inversores tipo híbridos, el sistema puede estar conectado o desconectado de la red según se requiera o desee, en este caso la red pasa a ser un sistema de emergencia (Akbarzadeh \& Victoria, 1992; SchneiderElectric, 2015; SchneiderElectric, 2012; Laird, 2013). La viabilidad económica fue alcanzada principalmente a través del remplazo del sistema para calentamiento de agua -duchas, cocina, jacuzzi, piscina-, usando pipetas de gas por energía solar, generando un ahorro suficiente para financiar la implementación del sistema fotovoltaico propuesto. La generación de energía de óptima calidad se traduce en un estado de comodidad y bienestar para el usuario; se reducen ineficiencias de operación en equipos eléctricos por lo tanto menos pérdidas energéticas en el consumo; se prolonga la vida útil de electrodomésticos ya que no se exponen a elevados periodos de drásticas fluctuaciones en tensión, frecuencia ni corriente (OCCIDENTE, 2004); se alcanza independencia energética debido a que la red deja de ser la fuente que suple electricidad a la casa (Rodríguez, 2008).

\section{Dimensionamiento sistema fotovoltaico}

Para el dimensionamiento del sistema y posterior selección de los equipos se aplica principalmente el proceso de diseño propuesto por Akbarzadeh (1994) y por Smets et al. (2016).

\section{A. Orientación de Paneles Fotovoltaicos}

El lugar donde se implementó el sistema, está ubicado en el municipio de Arbeláez, departamento de Cundinamarca, Colombia, con coordenadas geográficas $4.2728^{\circ}$ Norte, $74.4160^{\circ}$ Oeste. Posteriormente, se identificó el patrón del paso del sol durante un año, lo anterior permitió definir la orientación de las placas solares. A partir de la Figura 1, el paso del sol se presenta sobre el hemisferio sur durante los meses de diciembre, enero, febrero, marzo, septiembre, octubre, noviembre; y en el hemisferio norte en abril, mayo, junio, julio y agosto, lo anterior permite la posibilidad de fijar paneles orientados hacia el norte o sur (UO, 2007: UPME, 2005). 
(c) Univ: of Oregon SRML.

\$ponsor: BPA 7 : 7.42

Solar timo zone: -5

Colombla

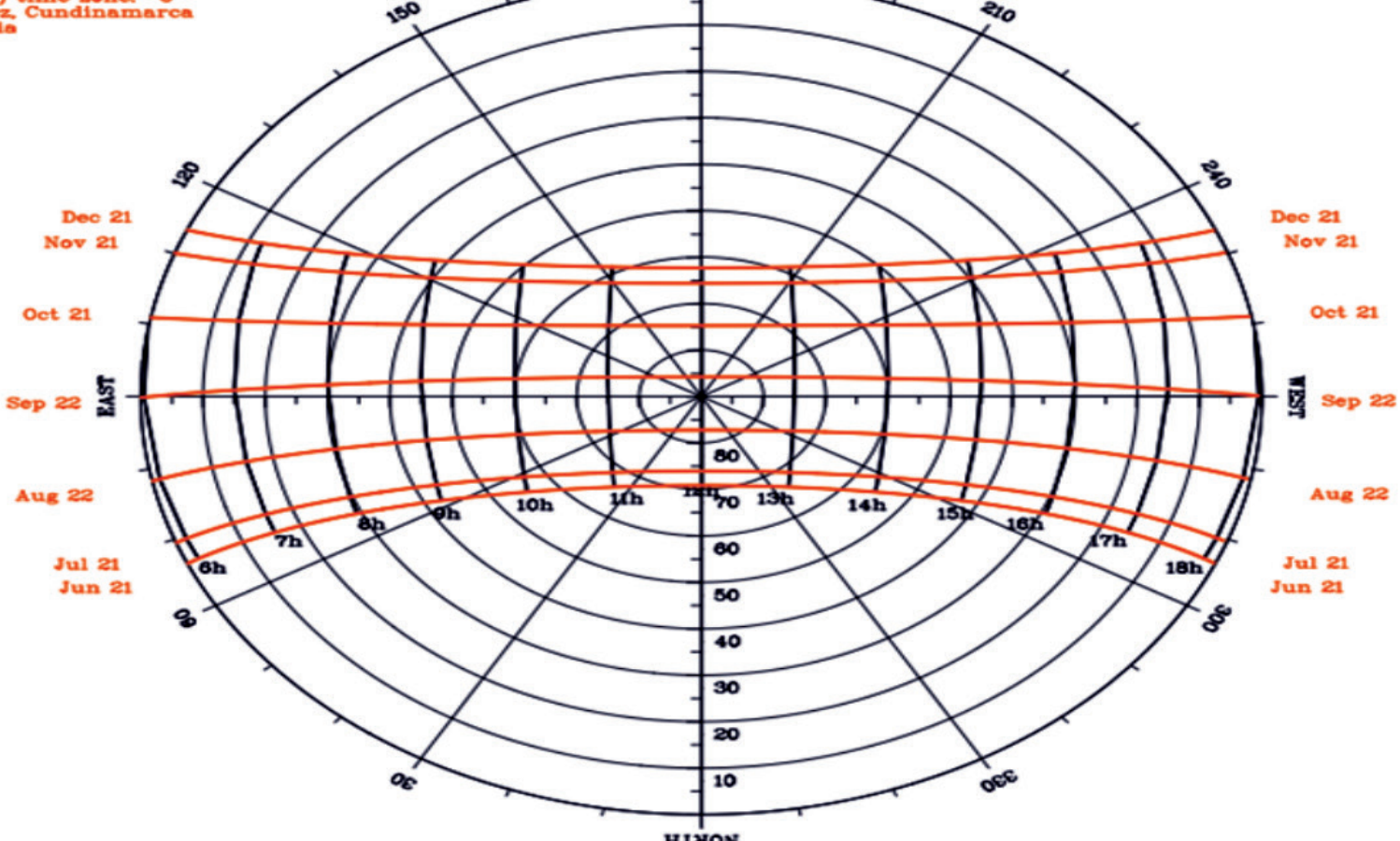

(c) Univ. of Orezon SRML.

Lat: 4.27: Long: -74.42

(Solar) time zone: $-\sigma$

Arbelaez Cundinamarca

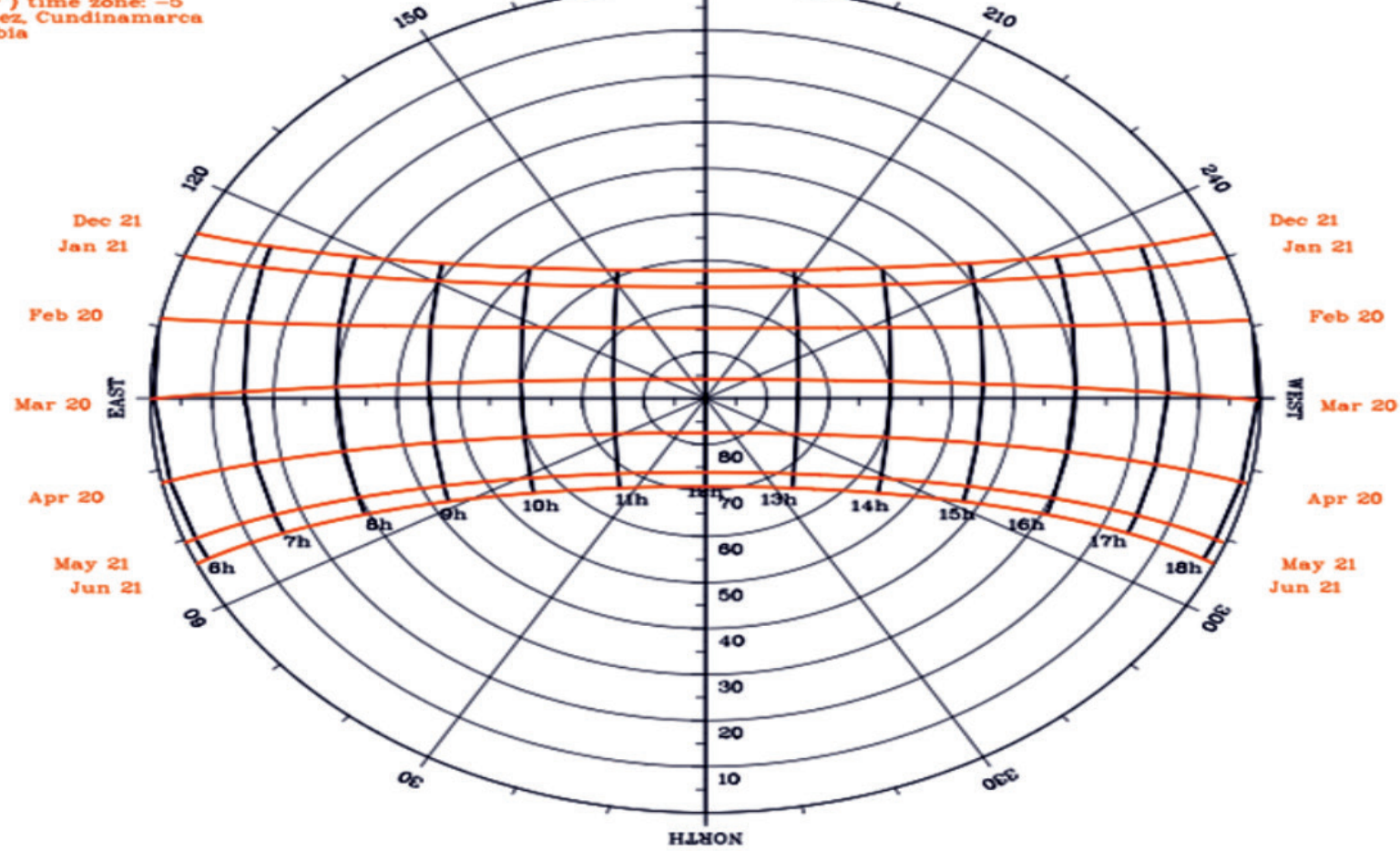

Estimated annual AC output:

Figura 1. Paso de sol (Sunpath) anual sobre Arbeláez, Cundinamarca.

Fuente: University of Oregon, 2016 


\section{B. Óptimo Ángulo de Inclinación, $\beta$}

Establecer el óptimo ángulo de inclinación ( $\beta$ ) de los paneles teniendo en cuenta la localización del proyecto (Figura 2) apunta a obtener la mayor generación de energía por área durante el funcionamiento del sistema, para hallar $\boldsymbol{\beta}$ se requiere obtener previamente el ángulo de declinación ( $\delta$ ) dependiente de la inclinación de la tierra con respecto al sol, este varía entre $23.45^{\circ}$ y $-23.45^{\circ}$ a lo largo del año. Inicialmente se calcula la posición de la tierra en la órbita $(\alpha)$ a partir de (1), luego los valores de $\delta$ a través de (2) y $\beta$ en (3) pueden ser calculados para cada día del año en el lugar del proyecto. Se determinó que el $\beta$ promedio anual es $14.94^{\circ}$ (Akbarzadeh \& Victoria, 1992); (Rodríguez \& Benítez, 2015); (Smets, Jäger, Isabella, Zeman, \& van Swaaij, 2016); (UPME, 2005).

$$
\alpha=\frac{2 \pi(n-1)}{365}
$$

Donde $\alpha$ es la posición de la tierra en la órbita en el $n$ día del año.

$$
\begin{gathered}
\delta=(0.006918-0.399912 \cos \alpha+0.070257 \operatorname{sen} \alpha-0.006758 \cos 2 \alpha+0.000907 \operatorname{sen} 2 \alpha \\
-0.002697 \cos 3 \alpha+0.00148 \operatorname{sen} 3 \alpha)\left(\frac{180}{\pi}\right)
\end{gathered}
$$

Donde $\delta$ es el ángulo de declinación solar con respecto a $\alpha$ de un día $n$ específico.

$\beta=\delta-L a t$

Donde $\beta$ se refiere al óptimo ángulo de inclinación geográfica dada según la localización del proyecto para las celdas solares en grados en la latitud (Lat) con el $\delta$ ángulo declinación solar previamente hallado con respecto al día del año evaluado.

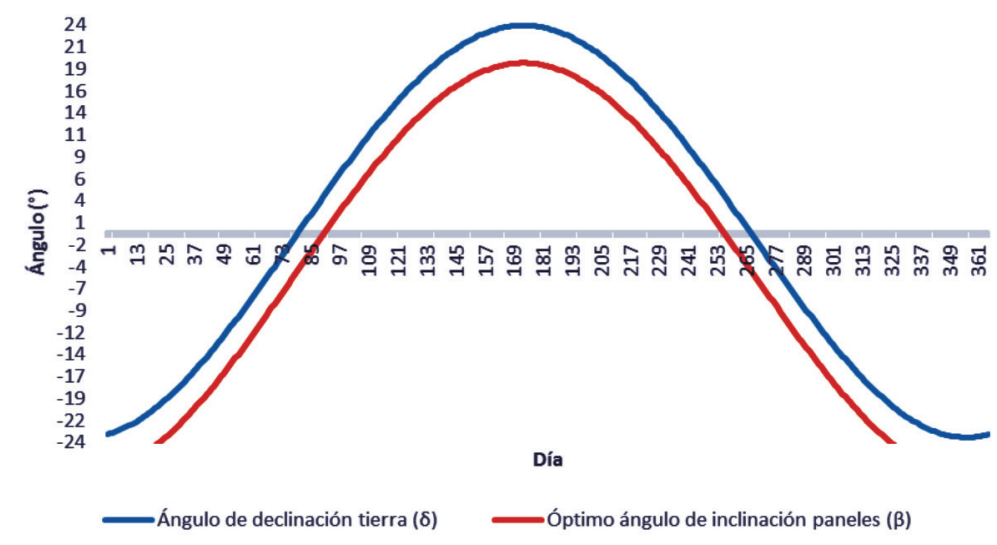

Figura 2. Óptimo ángulo de inclinación ( $\beta$ ) en Arbeláez, Cundinamarca. 


\section{Irradiación, temperatura y brillo solar sobre la superficie en el área del proyecto}

La irradiación sobre la horizontal y sobre planos inclinados contribuye a la evaluación de la generación energética que el sistema podría presentar (Akbarzadeh \& Victoria, 1992; Sonnenenergie, 2012; Smets et al., 2016). La Figura 3 compara la irradiación incidente sobre un plano y superficies inclinadas.
Es posible identificar el óptimo ángulo de inclinación, $\beta$, recibe una irradiación aproximada promedio de $4.91 \mathrm{kWh} \cdot \mathrm{m}^{-2} \cdot$ día- $^{-1}$; mientras que la superficie $\sin$ inclinación, la de $4^{\circ}$ y la de $19^{\circ}$ pueden experimentar irradiaciones promedio de $4.75 \mathrm{kWh} \cdot \mathrm{m}^{-2} \cdot \mathrm{dí}^{-}{ }^{-}$, $4.81 \mathrm{kWh} \cdot \mathrm{m}^{-2} \cdot \mathrm{día}^{-1}$ y $4.87 \mathrm{kWh} \cdot \mathrm{m}^{-2}{ }^{-}$día- $^{-1}$ respectivamente; evidenciando una potencial superior captación energética en caso $\beta$ sea usado.

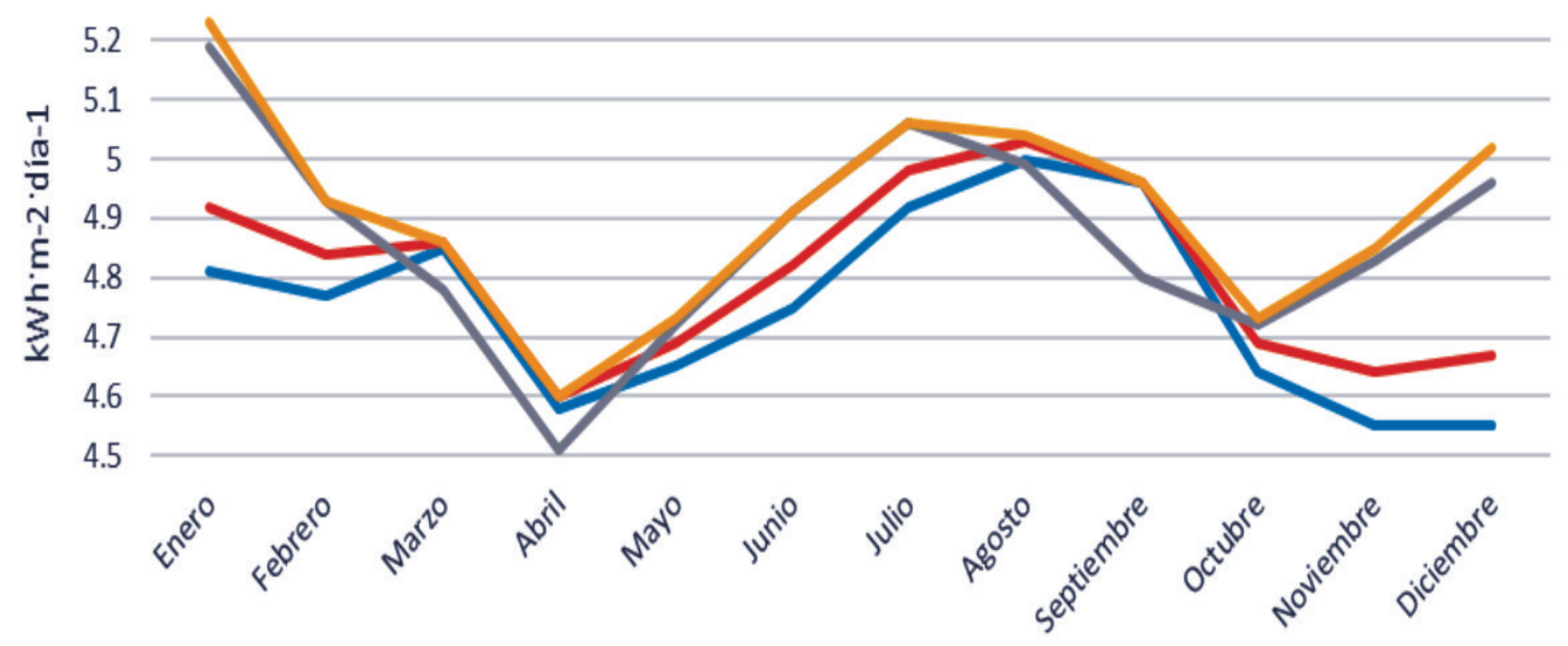

Meses

Incli. $0 \longrightarrow$ Incli. $4 \longrightarrow$ Incli. 19 Óptimo ángulo

Figura 3. Promedio de incidencia de radiación solar sobre superficies en Arbeláez, Cundinamarca, 2000-2005

Por otro lado, la temperatura del aire en el área a la altura disponible para fijar placas solares, presentada en la Figura 4, puede influenciar el adecuado proceso fotovoltaico de éstas. Las celdas requieren rangos de temperatura determinados por el fabricante para obtener un mayor rendimiento y no presenciar drásticas reducciones en la vida útil, por lo tanto, se hace fundamental evitar alcanzar el punto de estancamiento térmico -stagnation tempeture point- durante operación, ya que este estado decrementa la eficiencia del sistema (Akbarzadeh \& Victoria, 1992; Smets et al., 2016). Los módulos FV pueden reducir su eficiencia entre $0.3-0.4 \%$ por cada $1^{\circ} \mathrm{C}$ de incremento superior a las condiciones estándar de prueba, STC -Standar Test Conditions-, de los paneles que corresponde a $25^{\circ} \mathrm{C}$ y $1000 \mathrm{~W} \cdot \mathrm{m}-2$ (Schneider Electric, 2013; Nakagami et al., 2003; Ueda et al., 2009). Otro factor determinante, previo al dimensionamiento de un sistema fotovoltaico, es la cantidad de horas de sol por día disponibles, PSH -Peak Sun- Hours- en el sitio del proyecto, de acá se obtiene un estimado de la generación energética a partir de los paneles a ser instalados, para el lugar objeto de este trabajo es de cuatro (4) horas diarias promedio anual (Akbarzadeh \& Victoria, 1992; Rodríguez \& Benítez, 2015; Smets et al., 2016; UPME, 2005). 


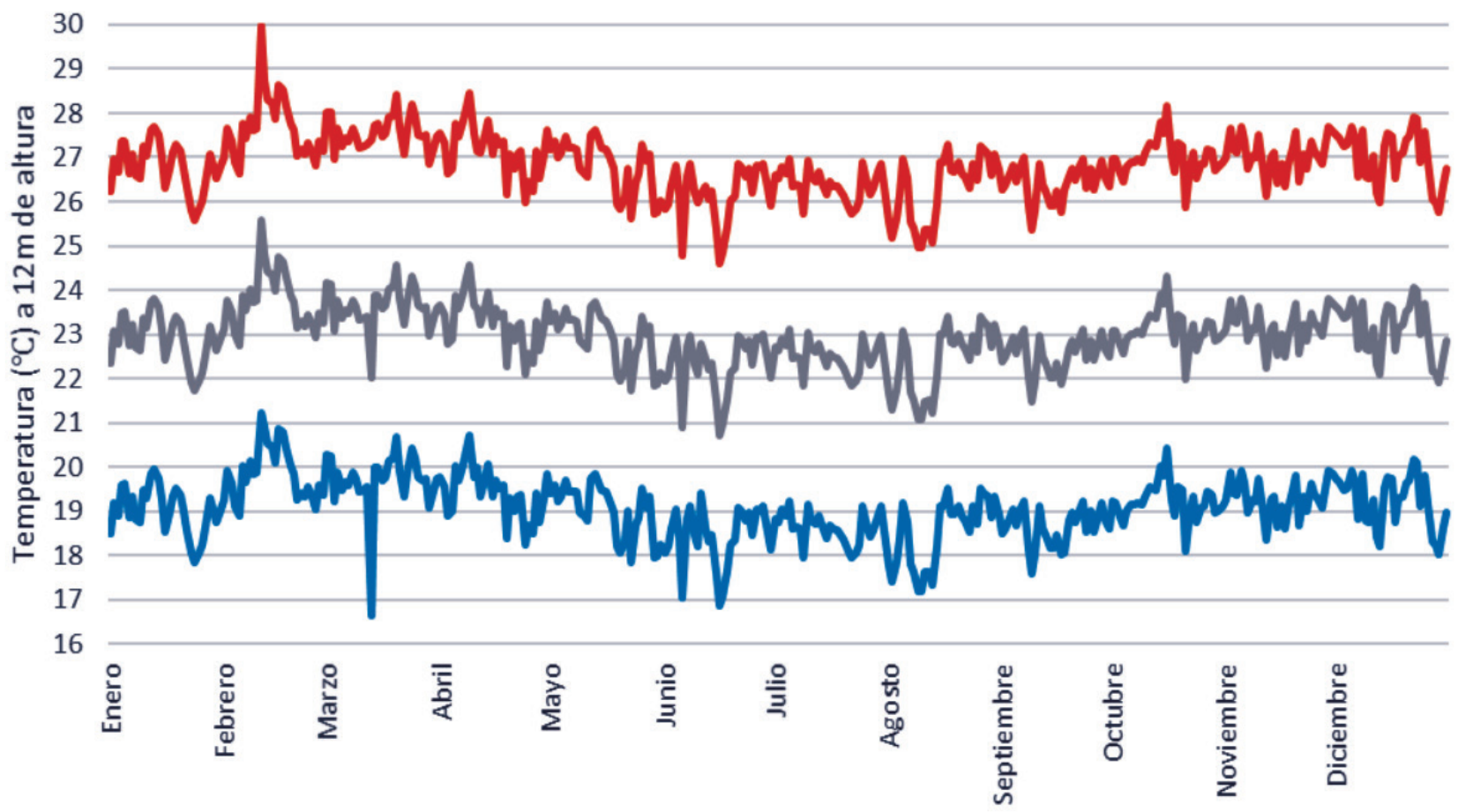

Figura 4. Temperaturas máximas, medias y mínimas anuales en Arbeláez, Cundinamarca Fuente: University of Oregon, 2016

\section{Demanda Energética}

Para determinar adecuadamente la demanda, se ajustaron hábitos de consumo promedios presentados en casas de recreo similares a la referente del proyecto, estos hábitos se relacionaron con los recibos de electricidad provistos debido a que las cargas no fueron reportadas satisfactoriamente. La demanda total mensual, Demmen a suplir por el sistema es de $575 \mathrm{kWh}$-máximo consumo mensual histórico reportado en facturas de energía durante el año 2014-, esta demanda se da durante 21 días por el hecho de ser casa de recreo.

1. Horas demanda por día, Hrdía según usuario son seis (6) horas

2. Demanda día, Demdía (6 horas) en kWh es de 27.38 DC

3. Demanda día corregida, Demdco aplicando un incremento del 10\% sobre Demdía para compensar pérdidas por captación, generación, transmisión, transformación y entrega energética del sistema (Smets et al., 2016); (Sonnenenergie, 2012) 30.12 kW AC por día

4. Demanda por hora, Demhr es 4.56 kWh AC

5. Las cargas a alimentar trabajan óptimamente a una tensión de 110V (AC) y frecuencia de $60 \mathrm{~Hz}$ de acuerdo a los requerimientos de la red eléctrica en Colombia (OCCIDENTE, 2004)

6. PSH significativas para generación energética (1000 W·m-2) por día a través de paneles fotovoltaicos en Arbeláez, Cundinamarca es de cuatro (4) horas (Rodríguez \& Benítez, 2015); (UPME, 2005); al determinar el número de horas de sol promedio por día en la zona del proyecto a partir de la irradiación anual promedio usando $\beta$ resulta en un valor de 4.91 PSH por día. Se selecciona un PSH de cuatro (4) para determinar el número de paneles a usar, es recomendable trabajar con el menos optimista escenario en el momento de diseñar. 


\section{E. Componentes Sistema FV}

La selección de las cantidades y componentes del sistema se hace a partir de la información presentada en las secciones anteriores. El sistema instalado en la investigación se programó para trabajar de forma híbrida, esto quiere decir que se usa la energía que viene de los paneles como fuente principal de energía para la casa y en caso que sea requerido y las baterías bajen su porcentaje de carga al 50\%, la red eléctrica entraría a trabajar y así no dejar la casa sin energía. En caso de que el inversor no permita la entrada de la red eléctrica se deberá a que el voltaje que ésta trae es muy alto, voltaje igual o superior a $130 \mathrm{~V}$ resulta en un riesgo para todos los equipos o electrodomésticos que se usan en la casa (OCCIDENTE, 2004); (Schneider Electric, 2015); en otras palabras el sistema está protegiendo los electrodomésticos de sufrir daños por altos voltajes y constantes fluctuaciones de la red eléctrica, si este problema no es solucionado, tarde o temprano los equipos serán afectados al igual que el inversor el cual tiene que trabajar con ese voltaje tan alto que sobrepasa los límites de trabajo fijados. El inversor permite pasar y trabajar con un máximo de $125 \mathrm{~V}$, si este voltaje es mayor, por seguridad el inversor no permite el paso de energía ni el uso de la red adecuadamente, cada vez que se presente una fluctuación en rangos mayores de $125 \mathrm{~V}$, el inversor marcará falla (Schneider Electric, 2015; Schneider Electric, 2012).

Cuando un equipo se inicia, en su arranque puede consumir desde dos (2) hasta incluso tres (3) veces su consumo nominal (Schneider Electric, 2012), este hecho es la principal razón de una posible sobrecarga del sistema FV, cuando se cuenta con una capacidad de transferencia de energía -capacidad de inversión-, muy cercana a la capacidad instalada total (Schneider Electric, 2015); (Schneider Electric, 2012).Los inversores seleccionados para el proyecto cuentan con $15 \%$ extra de capacidad sobre la demanda por hora reportada. La Figura 5 presenta el diagrama unifilar general del sistema FV propuesto.
Se utilizan paneles marca CanadianSolar referencia CS6P-255P Quartech con potencia nominal, PPS de $255 \mathrm{~W}$ y y eficiencia, $\eta$ de $15.85 \%$ de conversión de energía solar a energía eléctrica DC bajo STC. Estos a $25^{\circ} \mathrm{C}$ presentan su mayor rendimiento, pero en condiciones de temperaturas superiores a $47^{\circ} \mathrm{C}$ su funcionamiento normal puede verse afectado. Es importante mencionar tal como se evidencia en la Figura 4, el lugar no ofrece temperaturas fuera de los rangos de funcionamiento normal, indicados según ficha técnica (CanadianSolar, 2013). Para determinar la cantidad de paneles y por ende el tamaño del sistema FV en $\mathrm{kWp}$ requerido para suplir la demanda presentada por el usuario, se utiliza (4). El resultado obtenido es de 29.52 paneles, se aproxima a 30 paneles, ocupando un área de $49 \mathrm{~m} 2$ con un peso total de $555 \mathrm{~kg}$ que deberá soportar la cubierta.

$N P S=\frac{\left(D E M_{\left.d c 0^{-1000}\right)}\right.}{H S D} \cdot P P S^{-1}$

Donde NPS es igual a número de paneles solares, adimensional.

La cubierta disponible cuenta con una inclinación de $19^{\circ}$, los paneles son instalados con dicha inclinación sobre una estructura de aluminio (Figura 6) diseñada para el proyecto. La estructura sostiene los paneles sin perforarlos. Los paneles serán divididos en dos subsistemas, cada uno de 15 unidades, configurados en cinco (5) paralelos con tres (3) series cada uno, obteniendo una corriente de operación, Imp de 42.15A con voltaje de operación, Vmp de 90.6V por subsistema. Los dos subsistemas alimentarán dos controladores de carga solar.

\section{1). Paneles fotovoltaicos}



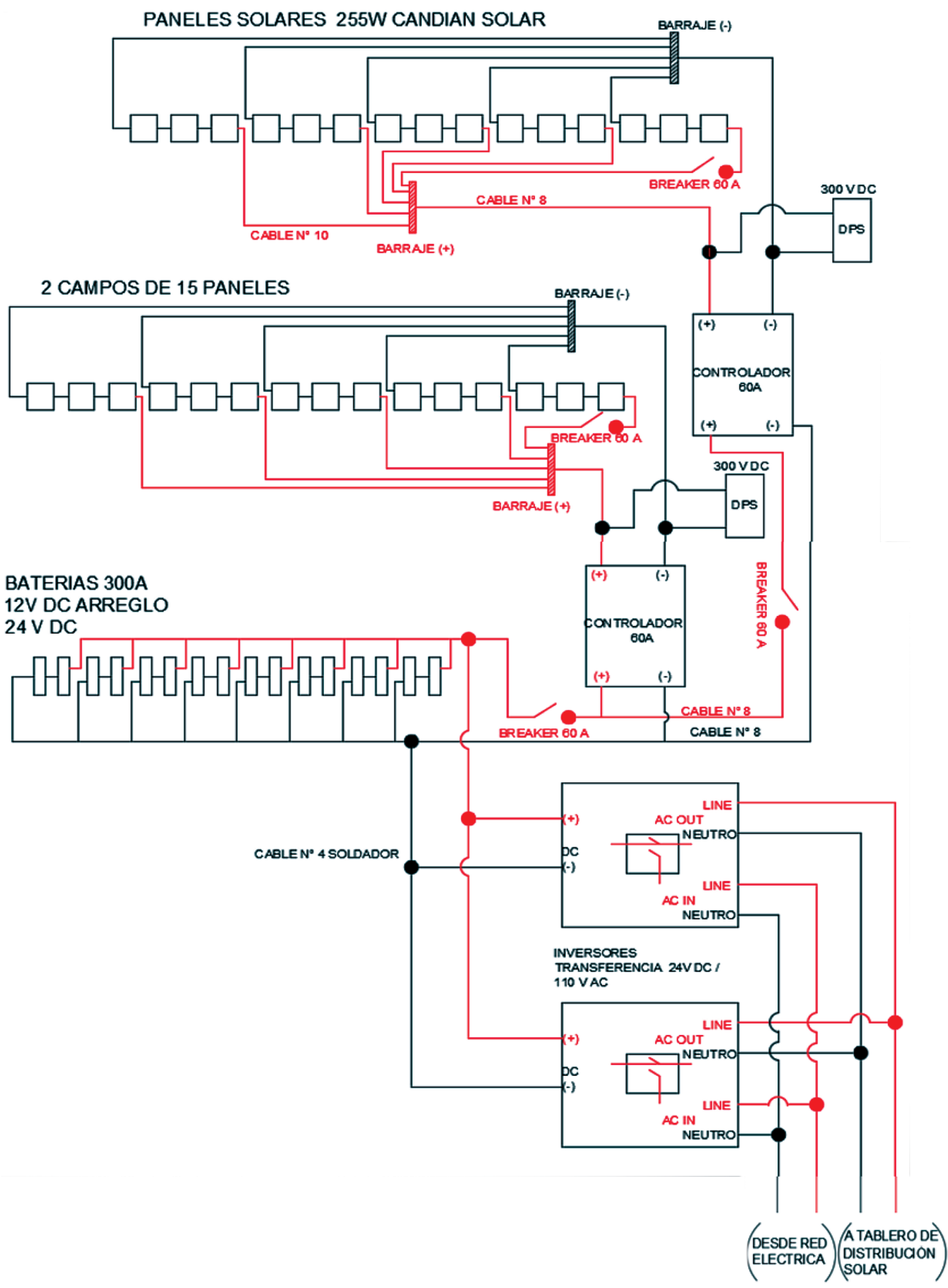

Figura 5. Diagrama unifilar sistema FV 


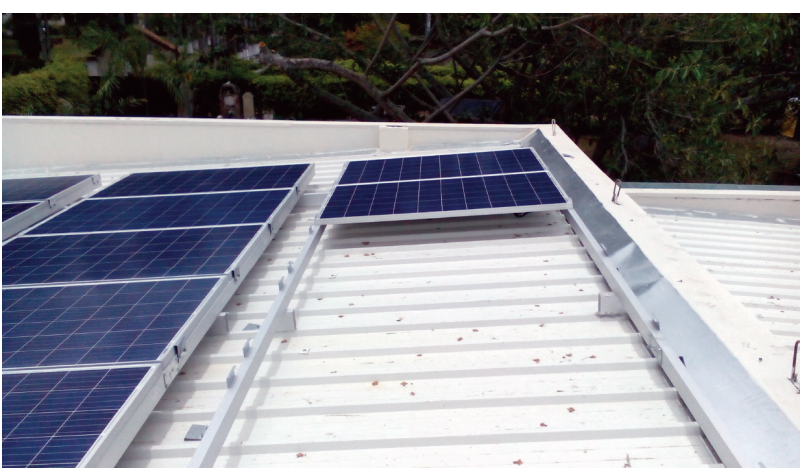

Figura 6. Estructura en aluminio para soportar paneles solares Fuente: El Autor

\section{2). Inversor}

Se seleccionan dos inversores/ cargadores tipo híbridos marca Schneider Electric referencia Conext SW 4024 120/240 con capacidad de potencia nominal de salida de $3400 \mathrm{~W}$ cada uno, trabajan desconectados o desconectados de la red según se requiera. Estos soportan la DEMhr AC en caso se presente, y garantizan que el sistema continúe operando, incluso en el momento de arranque de cargas simultáneamente cuando existe la posibilidad estas demanden hasta tres veces su potencia de consumo nominal, cada inversor cuenta con una capacidad de salida de 7000W durante cinco (5) segundos. Esta referencia permite la entrada y salida de DC desde y hacia un banco de baterías a $24 \mathrm{~V}$, también la entrada y salida de $A C$ desde y hacia la red eléctrica, para este proyecto se transforma energía a 120VAC con una frecuencia de $60 \mathrm{~Hz}$ (SchneiderElectric, 2012).

\section{3). Banco de baterías}

El banco de baterías es compuesto por 16 baterías tipo plomo-ácido marca MTEK referencia 12300 con capacidad de 300 Amp- hora (Ah) a 12VDC. Se asigna un \% profundidad de descarga, DOD del $50 \%$ buscando extender la vida útil de las baterías al máximo posible dentro del presupuesto establecido. Se acumula la Demdía, debido al DOD establecido, el número de baterías se duplica (5). El voltaje del banco de baterías es de $24 \mathrm{~V}$ en respuesta a limitaciones técnicas del inversor escogido, cada batería acumularía aproximadamente $3600 \mathrm{WhDC}$, todo el banco cuenta con cerca del $105 \%$ de la DEMdía.

$$
N B a=\frac{D E M_{d i a}}{((V d c \cdot A h) / 1000)} / D O D
$$

Siendo NBa el número de baterías.

Se conectan en serie y paralelo, ocho (8) líneas de dos (2) baterías, obteniendo 24VDC y 2400Ah.

\section{4). Controlador de carga solar}

Dos controladores de carga marca Schneider Electric de referencia Conext MPPT 60150 serán usados para el sistema como se observa en la Figura 7, los controladores que permiten el seguimiento del punto máximo de energía son denominados MPPT maximum power point tracking-, contribuyen a obtener la mayor cantidad posible de la energía captada por lo paneles, son responsables de cargar el banco de baterías según configuración de este. Cada uno soportará un subsistema, generan hasta 60 amperios de corriente de carga con una capacidad de hasta 150VDC provenientes de los paneles (Schneider Electric, 2013).

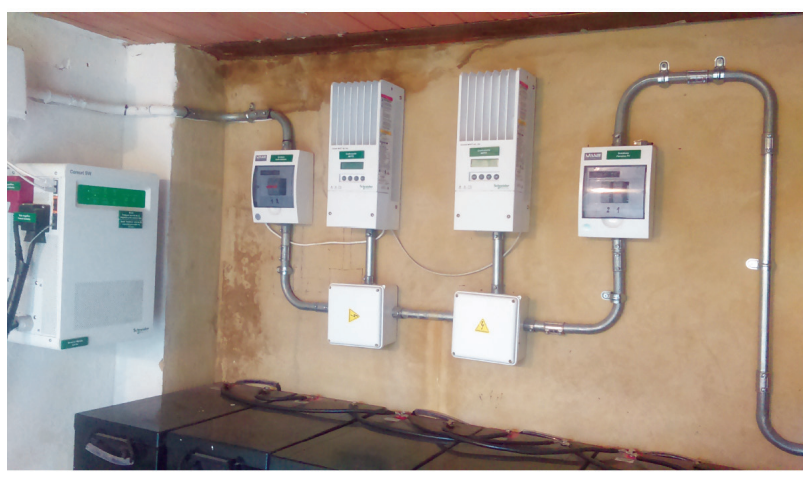

Figura 7. Vista general componentes del sistema

\section{5). Dispositivo para protección de rayos y so- bretensiones (DPS)o supresor de picos}

Se instalan dos dispositivos marca Midnite Solar referencia MNSPD300DC, uno por cada subsistema de paneles, cada supresor de picos puede manejar hasta 300V DC. Instalados dentro de cofres localizados en cubierta (Figura 8). 


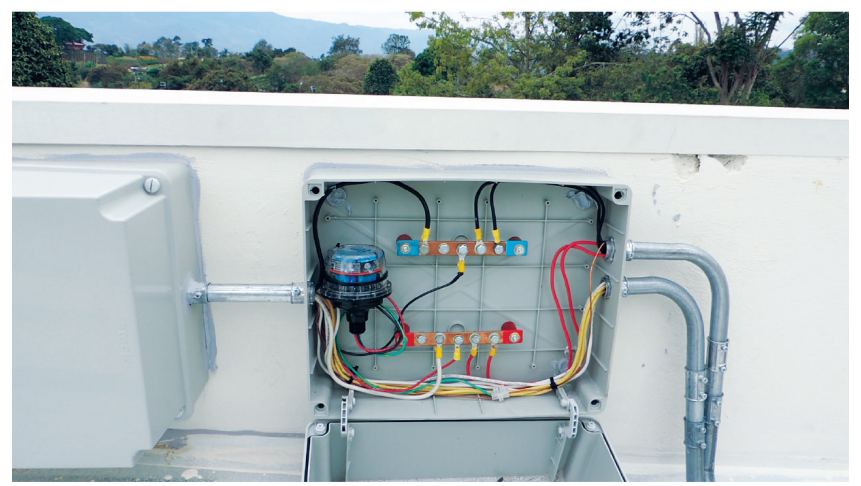

Figura 8. DPS Midnite Solar 300V DC

\section{6). Sistema de comunicación}

Estos dispositivos presentan información en tiempo real del estado del sistema, generación energética, voltajes por parte de la red y el sistema FV, demanda presentada, porcentaje carga del banco de baterías. Además, permiten configurar el sistema según requerimientos del usuario de forma remota o en el sitio donde se localizó el sistema. Se instala un panel de control marca
Schneider referencia SCP como se muestra en Figura 9, éste se compone de un monitor tipo LED de fácil lectura y presentación de datos en lugar de instalación. EI COMBOX, dispositivo con capacidad de enviar y recibir información del sistema vía internet, requiere de conexión local de internet. Los dos dispositivos conforman el sistema de comunicación (Schneider Electric, 2014a; Schneider Electric, 2014b).

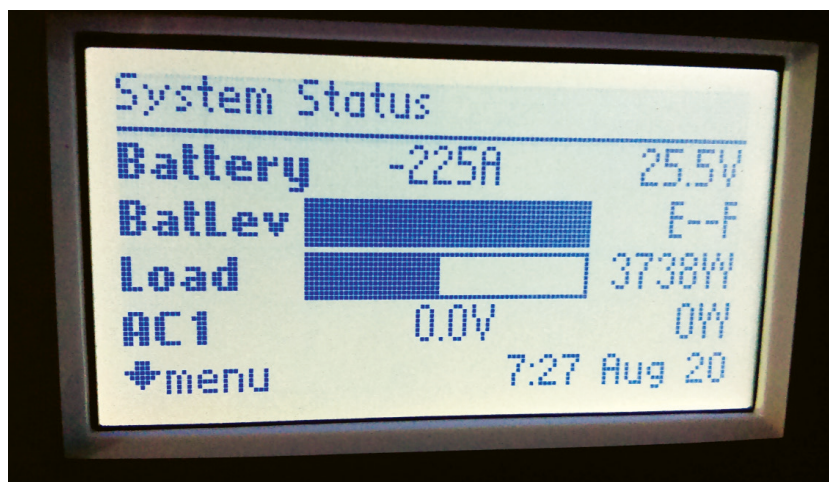

Figura 9. Datos presentados por SCP marca Schneider.

\section{Resultados y discusión}

El sistema fue diseñado para suplir DEMdco determinada a partir de las cargas presentes en el predio. Esta demanda abarca los equipos que trabajan a una sola fase $(120 \mathrm{~V} / 60 \mathrm{~Hz})$, equipos que trabajan en diferentes fases por lo general presentan grandes consumos y pueden agotar muy rápido las baterías y así reducir la vida útil del sistema fotovoltaico. El usuario cuenta con la posibilidad de desconectarse completamente de la red, además de generar energía de calidad para su uso. La generación real promedio del sistema mostrada en la Figura 10 es de $19.75 \mathrm{kWh}$ diarios, esto equivale al $66 \%$ de la demanda presentada por el usuario, con una producción energética promedio hora de 4.94kWp durante el tiempo que lleva operando el sistema. Lo anterior representa una reducción total 
desde diciembre de 2015 hasta agosto de 2016 de $982 \mathrm{~kg}$ de $\mathrm{CO}_{2 \mathrm{eq}}$ tal como lo refleja la Figura 11, con un factor de emisión de $0.18259 \mathrm{kgCO}_{2 \text { eq }}$ por cada kWh comprado de la red en el área. Cada kg de $\mathrm{CO}_{2 \text { eq }}$ no emitido representa el no aumento del calentamiento global, contribuyendo al desarrollo sostenible y a la mitigación de la contaminación atmosférica (Ecometrica, 2015).

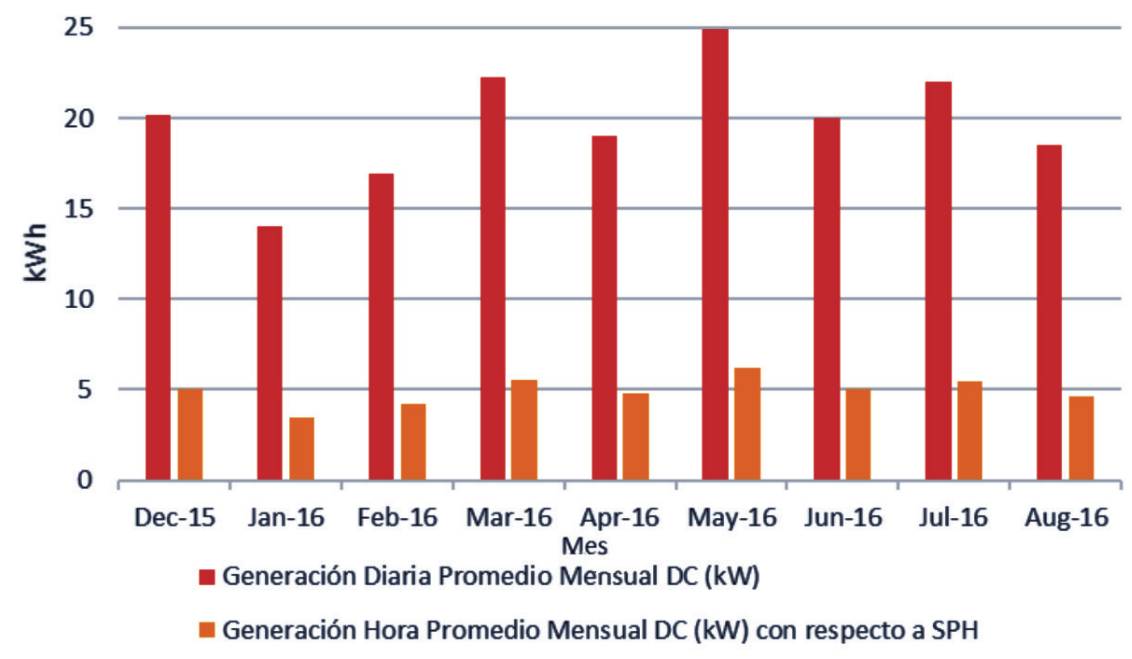

Figura 10. Generación real sistema fotovoltaico en Arbeláez, Cundinamarca

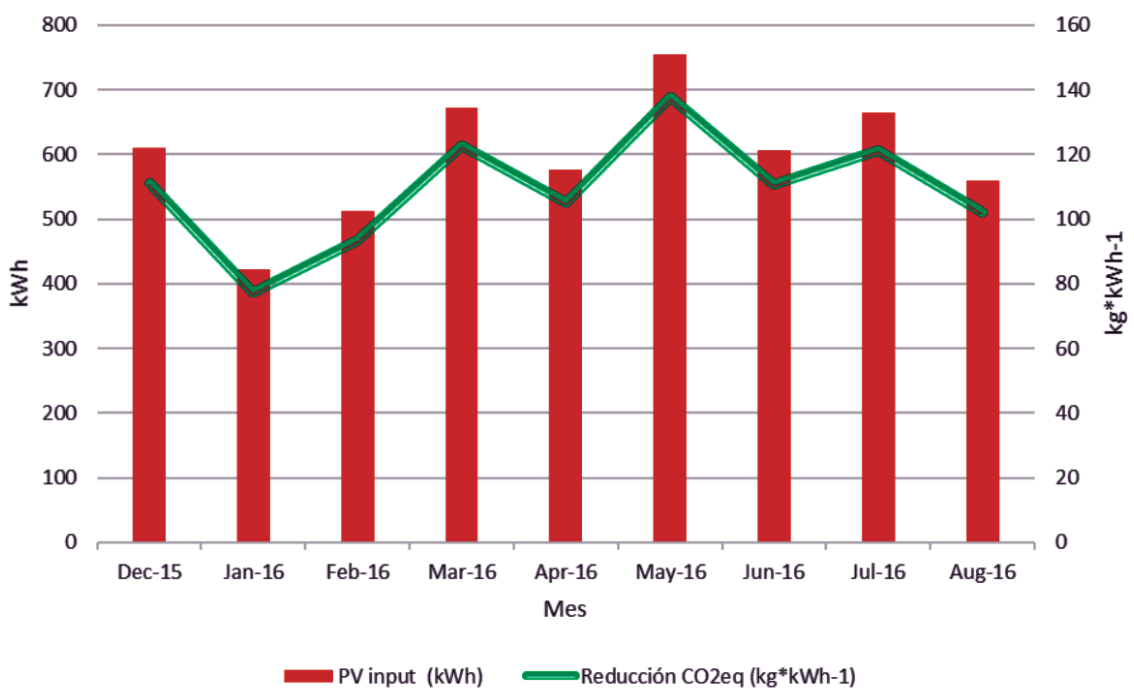

Figura 11. Generación FV en kWh en DC mensual- reducción emisiones de $\mathrm{CO}_{2 e q}$ ligada a electricidad ( $\mathrm{kWh}$ ) no comprada a la red eléctrica

La Figura 12, a partir de datos descargados del equipo COMBOX permite observar la generación energética en AC suple la demanda presentada por el usuario entre el periodo de diciembre de 2015 a agosto de 2016, con una leve caída en la producción en abril. El comportamiento del inversor uno -maestro-, y dos -esclavo-, responde a la configuración dada, el inversor maestro siempre está presente, en cambio el inversor esclavo entra como apoyo cuando la demanda requiere una inversión de $\mathrm{DC}$ a $\mathrm{AC}$ de por lo menos $1400 \mathrm{~W}$, que equivale al $20 \%$ de la capacidad nominal del sistema para transformar energía en adelante. 


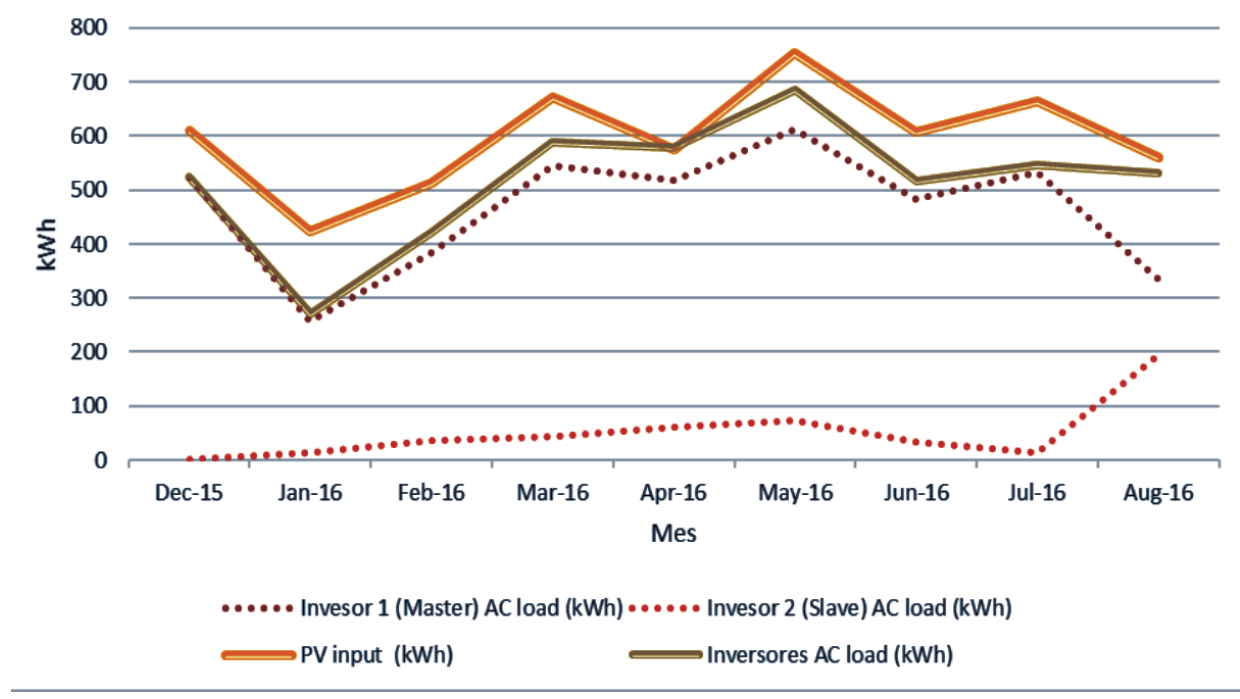

Figura 12. Generación energética AC Vs. Demanda energética AC

Los usuarios, buscando contar con energía eléctrica disponible cada vez que hagan uso del predio, inician una autoevaluación de su consumo y lo priorizan. Generalmente a las 15 horas revisan el porcentaje de carga de baterías, con esta información deciden cuánta más energía solar resta en el día para cargar baterías y que cargas deben evitar si es requerido y así pasar la noche completa con servicio energético sin uso de la red, teniendo sumo cuidado no descargar baterías por debajo de un DOD de 50\%. Desde el mes de junio de 2016 la demanda se presenta relativamente constante, la producción por parte del sistema FV es suficiente para suplir la demanda regulada que actualmente maneja el usuario, 17kWh día, eliminando la entrada del sistema eléctrico nacional. El uso de la red eléctrica es restringido únicamente a días que no presenten adecuada irradiación solar para cargar el banco de baterías, está entra automáticamente cuando el bando alcanza un DOD de $50 \%$ y sale cuando se alcanza un DOD de $60 \%$.

\section{Conclusiones}

La implementación de sistemas fotovoltaicos en zonas rurales del país es una alternativa viable a la red nacional; se traduce en bienestar del usuario, independencia energética, generación energía eléctrica de óptima calidad, incremento en la vida útil de electrodomésticos, conciencia de consumo por parte del usuario conduciendo a alcanzar eficiencia energética. La reducción de GEI por instalación de placas solares es significativa, por lo tanto, no se contribuye al calentamiento global que se experimenta en la actualidad. Es importante revisar y corroborar el incremento entre la demanda actual y la tomada como base de diseño, en caso de que se busque aumentar la cantidad de equipos que el sistema fotovoltaico debe alimentar.

La determinación de pérdidas en la captación, transmisión, acumulación, producción energética del sistema FV por equipos que hacen parte de este debe ser rigorosa. La información de irradiación solar y PSH siempre que sea posible, debería ser generada en campo.

La inclusión de sistemas de monitoreo en tiempo real permite al usuario conocer e identificar aspectos como porcentaje de carga de baterías, tensión de la red, tensión del sistema, generación eléctrica desde paneles, demanda eléctrica, entre otros. Incluso es posible monitorear, configurar, evaluar el sistema desde cualquier dispositivo con acceso a internet (Schneider Electric, 2014a, 2014b, 2015). 
A medida que el usuario se familiariza con el sistema, sus funciones e incluso limitaciones; es cuando los beneficios de ser eficiente energéticamente son evidenciados, se inicia un proceso de autoevaluación del consumo previo al sistema y el actual, se restringe el uso de equipos de acuerdo a su potencia y duración de su uso simultáneamente con otro equipo de características específicas que pueda afectar el adecuado funcionamiento del sistema -sobrecarga, agotamiento excesivo e injustificado del banco de baterías, sobre demanda en horarios nocturnos-.

\section{Literatura citada}

1. Akbarzadeh, A., \& Victoria, R. E. A. o. (1992). Fundamentals of Remote Area Power Supply Systems: A Text for Tertiary Students: Renewable Energy Authority of Victoria (Energy Victoria).

2. CanadianSolar. (2013). SOLAR MODULE, Installation Manual (UL). In C. Solar (Ed.).

3. Ecometrica. (2015). The Emission Factors. Retrieved from www.emissionfactors.com

4. Hou, G., Sun, H., Jiang, Z., Pan, Z., Wang, Y., Zhang, X., Yao, Q. et al. (2016). Life cycle assessment of grid-connected photovoltaic power generation from crystalline silicon solar modules in China. Applied Energy, 164, 882-890. doi: http://doi.org/10.1016/j.apenergy.2015.11.023

5. Laird, J. (2013). Inverters and beyond. Renewable Energy Focus, 14(3), 32-36. doi: http://dx.doi.org/10.1016/ S1755-0084(13)70052-8

6. Nakagami, H., Ishihara, O., Sakai, K. \& Tanaka, A. (2003). Performance of Residential PV System Under Actual Field Conditions in Western Part of Japan. (36762), 491-497. doi: 10.1115/ISEC2003-44228

7. OCCIDENTE, U. D. A. U. A. D. (2004). Calidad de la energía eléctrica. Retrieved from Colombia:

8. Plangklang, B., Thanomsat, N. \& Phuksamak, T. (2016). A verification analysis of power quality and energy yield of a large scale PV rooftop. Energy Reports, 2, 1-7. doi: http://dx.doi.org/10.1016/j.egyr.2015.12.002

9. Rodríguez, D. Á., M. \& Benítez, J. (2015). Solar Radiation Atlas for the region Cundiboyacense Colombia through radiometric data. 13th LACCEI Annual Intenational Conference. doi: http://dx.doi.org/10.18687/ LACCEI2015.1.1.142

10. Rodríguez Murcia, H. (2008). Desarrollo de la energía solar en Colombia y sus perspectivas. Revista de Ingeniería, 83-89.

11. Schneider Electric. (2012). INVERSOR/CARGADOR CONEXT SW 4024 (865-4024), Guía de Instalación. In S. Electric (Ed.), (Vol. 865-4024): Schneider Electric

12. Schneider Electric. (2013). CONTROLADOR DE CARGA SOLAR CONEXT MPPT 60 150, Instalación y Guía del Propietario. In S. Electric (Ed.): Schneider Electric

13. Schneider Electric. (2014a). CONEXT ComBox (8651058), Quick start guide. In S. Electric (Ed.), (Vol. 8651058): Schneider Electric

14. Schneider Electric. (2014b). CONEXT System Control Panel Installation Guide (865-1050-01). In S. Electric (Ed.), (Vol. 865-1050-01): Schneider Electric.

15. Schneider Electric. (2015). INVERSOR/CARGADOR CONEXT SW (976-0315-03-01), Actualización de la documentación. In S. Electric (Ed.), (Vol. 976-0315-0301): Schneider Electric.

16. Smets, A., Jäger, K., Isabella, O., Zeman, M. \& van Swaaij, R. (2016). Solar Energy: The Physics and Engineering of Photovoltaic Conversion, Technologies and Systems: UIT Cambridge.

17. Sonnenenergie, D. G. F. (2012). Planning and Installing Photovoltaic Systems: A Guide for Installers, Architects and Engineers: Taylor \& Francis.

18. Ueda, Y., Kurokawa, K., Kitamura, K., Yokota, M., Akanuma, K., \& Sugihara, H. (2009). Performance analysis of various system configurations on grid-connected residential PV systems. Solar Energy Materials and Solar Cells, 93(6-7), 945-949. doi: http://dx.doi.org/10.1016/j. solmat.2008.11.021

19. UO, T. U. o. O. (2007). Solar Radiation Monitoring Laboratory.

20. UPME. (2005). Atlas de Radiación Solar de Colombia. Retrieved from Bogotá, Colombia.
Conflicto de Intereses

Los autores declaran no tener ningún conflicto de intereses

Recibido: octubre 10 de 2016 Aceptado: noviembre 04 de 2016 